\title{
Objects Control for Software Configuration Management
}

\author{
Jacky Estublier \\ LSR, Grenoble University \\ 220 Rue de la Chimie, BP 53 \\ 38041 Grenoble 9 France \\ Jacky.Estublier@imag.fr
}

\begin{abstract}
A major requirement in Software Engineering is to reduce the time to market. This requirement along with a demand for product sophistication and better quality has led to larger teams which in turn dramatically increases the pressure for more concurrent work in a distributed context.

This paper, based on our experience in Software Configuration Management for large software systems, shows why object management in such a context requires specific facilities for the consistent management of objects in multiple copies, different locations and formats, accessed and changed simultaneously by many engineers.
\end{abstract}

We present the solutions we have developed with our partner Dassault Systèmes, for the definition and enforcement of consistent concurrent engineering work, including a number of measures showing that scalability and efficiency are really tough issues.

We argue that the scalability and efficiency constraints found in SCM can only be met by a new architecture of SCM systems and by the development of a middleware layer that should be common to all SCM tools, and also usable by other applications sharing the same concerns.

Keywords: Software configuration management, Version control, concurrent engineering, distribution, architecture.

\section{Introduction}

The strongest driving force in industry is the reduction of the time to market. In software engineering, the life cycle (the delay between two releases of a software product), has tended to decrease from 18 months to 4 months. Simultaneously, the size and complexity of the developed product substantially increase, as well as the required quality for these products.

Coping with these conflicting characteristics requires an increase in the number of persons involved in the teams, and an increase in the degree of concurrency. Today, one of the major CAE issues is concurrent engineering control. As will be shown, concurrency engineering control requires different characteristics from the underlying infrastructure, not available today. 
The work presented here has been carried out in the area of software engineering, and more specifically in Software Configuration Management (SCM), but we believe that most, if not all, of our findings apply to all of CAE.

We will exemplify both the problem and the solution we propose with the experience we gained in addressing configuration management issues with our industrial partner Dassault Systèmes (DS). DS is the world wide leader in CAD/CAM/PDMII with its main software product CATIA. The fact that CATIA has over 4 millions lines and that it is developed simultaneously by 800 engineers with a 4 month life cycle, makes DS one of the world's major software producers. The numbers provided in this paper are measurements made on the real DS software.

Chapter 3 presents the issues, along with some illustrating measures, and shows that current technology does not satisfy our requirements. Chapter 4 presents our layered architecture; chapters 5, 6, 7 present each layer: Basic workspace manager, Synchronization manager and Concurrent Engineering manager

\section{Object Management in Concurrent Engineering}

The experience shows that we are a long way from the time when an engineer "owned" all the required objects, for the whole duration of an activity. Instead, at DS, each software engineer has direct access to all the needed objects; but due to the high degree of concurrency, each object is used by 50 to 100 different engineers simultaneously. Of course, changed objects must be kept private for the work duration. If $\mathrm{N}$ engineers change the same file concurrently, that file will have $\mathrm{N}+1$ different values (also called cooperative versions ). At DS, $\mathrm{N}=3$ in average $^{1}$; values for $\mathrm{N}$ greater than 6 are not uncommon, with maximum around 30 .

A high degree of concurrency requires two or more persons to access AND change the same piece of information in a concurrent way. The database community addressed and solved that issue long ago, using the transaction concept. Unfortunately, the typical database transaction lasts a fraction of a second and involves few objects, while in design, the time span of a change is the task that leads to that change i.e. from a few hours to a few weeks or more; and the scope of a change can be huge (thousands of objects). As a consequence, the database serializability approach (changes are performed in sequence) cannot be used here. Concurrent engineering requires engineers to work and change different copies of the objects of interest.

As engineering is predominantly human-driven, the intellectual atom of change is the task (or activity), i.e. a significant body of changes/creations leading to a new "consistent" state of the product. In the meantime, the state of the object is "inconsistent", and the engineer requires his/ her job to be isolated from the other changes. Each engineer needs private copies of the objects. Conversely, the engineer knows that

\footnotetext{
1 Averages are between 1 and 2 for kernel files, and around 3 for application modules
} 
other changes are concurrently performed on the same objects, and asks for these changes to be incorporated when relevant.

In CAE, activities are performed using tools. These tools have been designed to work on "objects" (often files) in a predefined format and organization. Depending on the activity, the same object may need to be found in a different format and location.

Apparently files not to be changed can be shared, at least on a LAN; experience shows this is not realistic for three reasons:

- Efficiency. At DS, with 1500 machines, average sharing of 30 and high performance demand (compilations), only local copies of all files can provide enough efficiency.

- Name and directory. The same file can be located under different names and directories depending on the platform (NT or Unix) or product version (restructuring).

- Internal format. Some files need to be translated from a format to another depending on the tool and activity which use it.

These reasons means that each file, even when not changed, has to be physically copied to the machine and workspace where it is used. At DS, at any point in time, each atomic object (e.g. a source file) has between 10 and 100 physical copies; in each case with potential read and write privileges.

Altogether the DS software amounts close to one million files, each averaging 30 copies (thus about 30 millions files) not necessarily all identical. In addition complexity, reliability (source code cannot be lost) and efficiency are really critical issues. Object management in such a context raises many difficult issues.

Altogether, CAE requires objects to be:

- in multiples copies, locations and formats,

- resynchronised when found relevant, with

- high efficiency, reliability and availability.

Providing a solution to the first point can be simple; the challenge is to provide also ways to solve the last two points.

The issue for an SCM or a SE platform designer is to find a technology upon which the environment can be built. In practice we need database for storing the information and middleware to handle distribution.

\section{Architecture}

All SCMs to date are built following the same architecture: a central database contains all the objects and their versions, using the same schema (definition). All workspaces (a file tree in a file system) share the same database. The structure and content of a (complex) object in a WS is always the same (see Fig 1) 


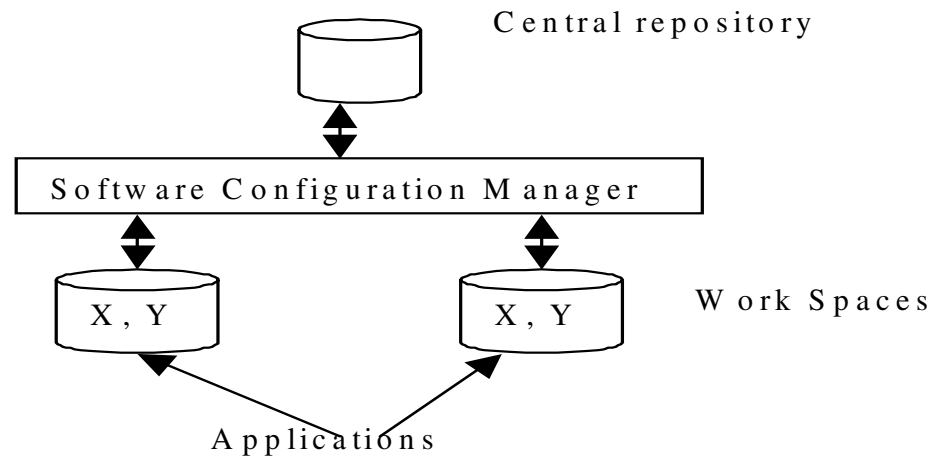

Fig. 1. Usual SCM Architecture

The first lesson learned in managing large software projects is that no central database can afford the needed efficiency and availability, even in the context of a LAN. For exemple, DS has an average of 2000 files per workspace. Synchronization operations involve on average a few hundred files i.e. a few MegaByte of information to transfer, merge, compress, format and so on. Consistency requires such heavy operations to be performed in transaction, but such transactions last several minutes during which objects are locked, and the server busy.

With an average of about 800 workspaces at any point in time, and such heavy transactions occurring on average 4 times a day per workspace (and most of them executed at about the same time i.e. on arriving at and leaving work), no DB can afford the required efficiency. Moreover, the measurements we took have shown that, even using a 100MB LAN, a transaction takes twice as long if the database and the WS are on different machines. Finally, availability requires that the whole company will not be stopped if a disk, a machine or the network crashes.

A major objective of this work was to find a truly distributed architecture, in which no repository is the main one, no one owns objects; each only contains object values. The satisfaction of the requirements also implies that we should be able to use different kind of repositories simultaneously (file systems and databases), with different schemas and on different kind of platforms (heterogeneity).

Availability, flexibility and efficiency all push us to consider as atomic (and often on the same machine): a WS, its manager and its associated repository. The architecture (Figure ) we propose has as its basic element a workspace manager, organized in three layers. The first one is the basic Work Space manager (shaded). It deals with the problem of providing to each user (and his/her tools) the needed objects at the current location and in the right format. The second layer provides basic functions for transferring / synchronizing objects between any two workspaces. The third layer provides concepts for the consistency of concurrent activities, for structuring the workspaces, and for defining and enforcing cooperative work policies which satisfy some consistency requirements (see Fig 2). A fourth layer, not presented here, deals with general process support; see [1][5][6][19][20]. 


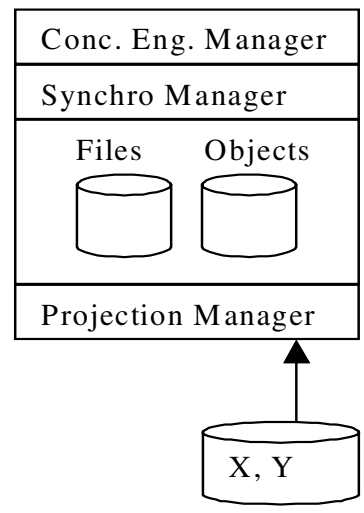

Repository and Representation for Application 1

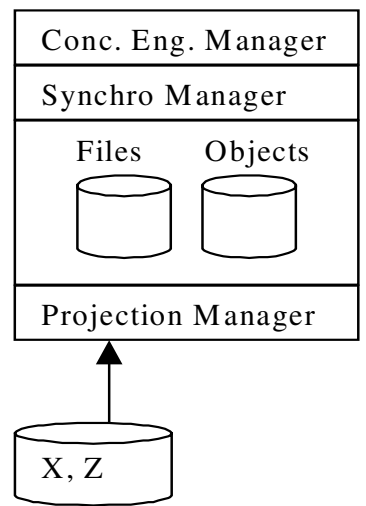

Repository and

Representation for

Application 2

Application 3

Fig. 2. Proposed Architecture

An object is an instance of a class in which the content and the behavior of each instance is defined. An object contains attributes. Attribute domains can be literals (i.e. strings, integers, file), or another object type; in the latter case we say the attribute is a composition attribute, and the object a complex or composed object.

This usual definition makes the identity: 1 object $==1$ instance. This definition was extended by the concept of revision: an instance containing the snapshot of an object at a given point in time ${ }^{2}$. This is not sufficent; in our case, at each point in time, an object may have many copies (different instances) that may have different values simultaneously, and even different definitions. We need to refine these definitions.

A Workspace (WS) is a sub-set of a public repository ${ }^{3}$ in which (part of) the objects of interest are stored.

A working object value is an object instance found in a WS. A object may simultaneously have different working values; one in each WS which contains it; but a WS can have one working value for a given object at the most.

2 The concept of revision is more complex; many aspects are not discussed here. Some attributes in a revision are immutable (like files), others are common to a set of revision, others are mutable; still other attributes trigger the creation of a new revision on the attempt to change them and so on. A few more details are presented later

3 A repository is a system which can store persistent information. It can be a file system or any kind of DB. It is public if access is performed by tools and users using the native repository functions without any need to be wrapped. It does not prohibit the control of these accesses, nor prevent undesired access, as long as this is done in a transparent way. 
We call representation the format of an object instance at a given location. An object may have many different representations. A revision is a snapshot of a working value, in the WS manager specific representation, or a copy of another revision.

Revisions represent snapshots of an object's value both at a different point in time and in different places (workspaces).

An abstract object, or simply an object, is the sum of all its instances i.e. all its revisions plus its working values.

As defined in [10], three levels of versioning apply to each object: Historical versioning which is represented by a sequence of revisions in a WS (often called a branch). Cooperative versioning represented by the different branches of an object (one by WS) and logical versioning, represented by variants which are different objects sharing some logical or historical properties.

This paper discusses the different issues raised by the management of cooperatives versions of an object.

\section{Basic WS Manager}

The fundamental goal of the basic WS manager is to provide the objects in the representation required by the tools working in this WS, irrespective of the other possible representations, and let applications change the object in that specific format, (apparently) irrespectively of the changes made by other applications on other working values of that object.

A WS manager manages two areas. The first one is the public WS i.e. a part of a public repository containing the working values; the other one is the private WS area containing revisions. The private WS area is intended to contain the information required for the management of the public WS.

A WS type is defined by:

- Object type definitions, for objects allowed to be contained in this WS.

- Repository type (Unix FS, NTFS, Oracle, etc).

- Projection functions. They define the relationship between the working value and revisions of an object

For each type of object, the following projection functions must be provided:

- Projection: A function defining the mapping from a revision to a working value. In general it is a partial function; some attributes may not be mapped, others can be added. Example: attribute souceCode of object foo of type c, is mapped to file foo.c; attribute owner is mapped as the owner of the file, attribute protection is added.

- Reverse projection: A function defining the mapping from a working value to a revision. If the public repository data model is weaker than the private 
one, this function requires conventions or additional information. Example: File foo.c is attribute sourceCode of object foo. But how are we to know that foo is a component of object $\mathrm{X}$ ?.

- Change mapper: A function defining the mapping from a working value change to a revision change. If the public repository data model is weaker than the private one, this function, in theory, cannot exist. This function usually requires conventions and heuristics or changes to be performed through a specific interface. Example: If file foo.c is renamed or moved into another directory, what does that mean for object foo?

This contrasts with all approaches we know, including SCM systems, where the representation is unique. Existing SCM systems support a single WS type where these functions are predefined. Either there is no object model at all, thus the private model is the public repository model, or conventions are simple enough to compute the functions trivially. For example name identity (object foo of type c maps to file foo.c); a single composition relationship mapped into the relationship between directory and file (e.g. if foo.c is under directory $\mathrm{X}$ then object foo is a component of object $\mathrm{X}$ ). The direct consequence is that (1) the object models are poor, and (2) WS type is unique[11].

In our system, WS types are formally defined, and projection functions are part of the type definition of each object type. For example, WS types proposed by SCM vendors can be defined easily which allows any product managed under "any" current SCM system to be integrated. It also provides for linking the work done on a product under an SCM system with the work done on the same product, under another representation, by another SCM system. This way we aim to address the virtual enterprise problem.

This approach is in complete opposition with current work in SCM (as well as our own previous work [9][11] ) on at least the following:

- There is no longer any central repository. Experience has shown that no centralized approach can scale to very large projects (like the Dassault Systèmes one).

- There is no generic WS manager, because there is no generic projection function, and because efficiency requires the WS manager to be tailored to benefit from current company conventions.

- Basic WS managers are indeed low-cost simple SCM systems. They can be compatible with current habits and simple revision control tools like RCS or CVS.

Basic WS managers can be simple, but unlike RCS or CVS, our solution is scalable toward high functionality levels, huge software products and very large distributed teams. This is the topic of the next chapters. 


\section{Synchronization Manager}

The synchronization manager defines how an object instance can be transferred and "synchronized" between two workspaces. Concurrent changes imply there is a way to reconcile different values of an attribute. We call that function the merge function. If we denote $\mathrm{A} 1, \ldots$ Ai the different values of attribute $\mathrm{A}$, and $\mathrm{Ai}=\mathrm{Ci}(\mathrm{A} 0)$ the value of $\mathrm{A}$ after change $\mathrm{Ci}$ is performed on $\mathrm{A} 0$, then a merge function $\mathrm{M}$ for $\mathrm{A}$ is such that $\mathrm{M}(\mathrm{Ai}, \mathrm{Aj})=$ $\mathrm{Ci}(\mathrm{Cj}(\mathrm{A} 0))=\mathrm{Cj}(\mathrm{Ci}(\mathrm{A} 0))=\mathrm{M}(\mathrm{Aj}, \mathrm{Ai})$.

This means the result of the merge is the same as if changes $\mathrm{Ci}$ and $\mathrm{Cj}$ were performed in sequence on A0, irrespective of the order. If an exact merge function existed for each attribute, concurrent engineering would always lead to consistent results! Unfortunately, for a given attribute, such a merge function either (1) exists, (2) is an approximation, or (3) does not exist at all. In our example, the components attribute has an exact merge, sourceFile merge is an approximate function, owner has no merge[15][16].

This approach contrasts with current work in SCM, at least with respect to the following aspects:

- Each WS manager ignores the formats and models used by the other WS managers; SCM system heterogeneity is possible.

- Only the relevant information is transferred, which is critical in distributed work (at DS, some complex objects total several Gigabytes!).

- We provide object merge instead of only file merge. Our customers are unanimous in saying that object merge is a major enhancement (the composition relationship merge is an exact merge).

\section{Concurrent Engineering Manager}

So far, nothing prevents us from to performing changes on any objects in each WS. Thus at a given point in time, each attribute of each object may have a different value in each WS in which it is present (at DS, each attribute would have 30 different values on average)!

Concurrent engineering control means ensuring that work performed collectively is "consistent". Unfortunately, for concurrent access to information, there is a single real consistency criteria: serializability (ACID transactions as found in databases) but is this case, concurrent changes are prohibited.

To which extent concurrent changes to an object are a relevant issue? A few measurements taken at DS answer the question. At DS, the average number of different values for a given file under work is around 3, with a maximum around 30.

Merges occur frequently, depending on the policy (see below) and kind of software. The average for an application file is 2 merges a year, for a kernel file 0.4 a year. These numbers may seem low, but averages are meaningless because most files are not 
changed or merged at all. Conversely, files under work are subject to many changes and merges. We have records of more than 200 merges a year for the same file, which means about 1 merge per work day. Globally, about one thousand merges occur each day at DS. Concurrent change control is a real critical issue.

These values apply only to files whereas, in this work and at DS, we deal with objects (files are atomic attributes in our object model). Our experience shows that concurrent changes to the same attribute (like file or composition) as well as changes to different attributes of the same object (like responsible, state, name, namefile, protection etc.) are very common. Merging must address both cases. For example, restructuring, renaming and changing files are common, independent activities. Raising the granularity from file to object makes appear new kinds of concurrent changes, which may produce new kinds of merges (typically composition changes). It is our claim that object concurrent change control subsumes traditional file control and provides homogeneous and elegant solutions to many difficulties which currently hamper concurrent software engineering.

\subsection{The Group Approach}

We call a group, a set of Work Spaces the goal of which is to make an object evolve in a "consistent" way. Each group contains a WS playing the role of reference repository for the group called the integration WS.

For consistency to be enforced in a group, the integration WS must behave as if ACID transactions are applied to it, with each WS playing the role of a local transaction cache.

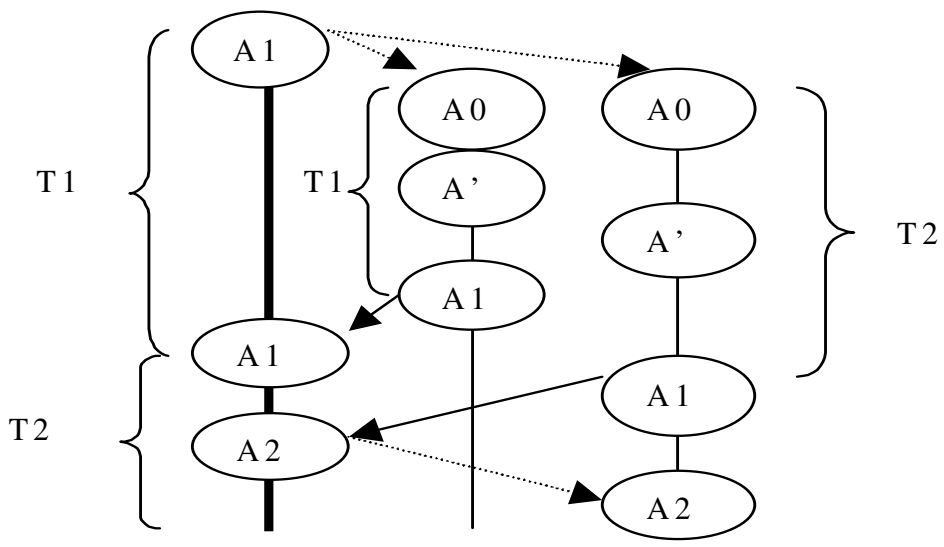

Fig. 3. Consistent Concurrent Changes

In Fig 3, both transactions $\mathrm{T} 1$ and $\mathrm{T} 2$ started with a copy of I/A0. Transaction T1 executed on I/A0 yields value W1/A1, while transaction T2 also performed on I/A0 yields value W2/A1. Changes T1 and T2 are consistent only if it is possible to compute the value I/A 2 as the result of applying T2 changes to I/A1 instead of I/A0.

If this is possible, the group $\mathrm{G}=(\mathrm{I}, \mathrm{W} 1, \mathrm{~W} 2)$ behaves as if all changes (T1, T2...) are applied in sequence on I in any order; while they are really performed concurrently on the different WSs of that group. 
Let us call $\mathrm{Wi} / \mathrm{O}$ and $\mathrm{Wi} / \mathrm{O}$.A the working values of object $\mathrm{O}$ and attribute $\mathrm{A}$ of $\mathrm{O}$ in WS Wi. We have defined the following atomic functions:

Propose. $\mathrm{P}(\mathrm{Wi} / \mathrm{O})$ informs that the current value for $\mathrm{O}$ in $\mathrm{Wi}$ is available to become the current value of the group.

Integrate. $\mathrm{I}(\mathrm{Wi} / \mathrm{O})$ performs $\mathrm{I} / \mathrm{O}=\mathrm{M}(\mathrm{I} / \mathrm{O}, \mathrm{Wi} / \mathrm{O})$.

Synchronize. $\mathrm{S}(\mathrm{O})$, when performed by $\mathrm{Wi}$ does $\mathrm{Wi} / \mathrm{O}=\mathrm{M}(\mathrm{Wi} / \mathrm{O}, \mathrm{I} / \mathrm{O})$.

Reserve and free. $\mathrm{R}(\mathrm{O} . \mathrm{A})$ and $\mathrm{f}(\mathrm{O} . \mathrm{A})$. $\mathrm{R}(\mathrm{O}$.A) sets a lock on O.A for the whole group and $\mathrm{f}(\mathrm{O} . \mathrm{A})$ releases that lock. Together they define a critical section for attribute O.A. At any point in time, a single WS of the group can be in the critical section for attribute A.

Free $(\mathrm{O})$, releases all pending locks on attributes of $\mathrm{O}$ in that group.

\subsection{Basic Policies in a Group}

A basic concurrent engineering policy means defining and enforcing

- who has the right to perform a change on what (not anyone on anything at any time),

- what is to be merged and when,

- who has to merge.

Experience shows that merge control (including merge prohibition) is a central point when the merge function is missing or approximate. More specifically, file merge is an approximation which requires, potentially, manual work (for conflicts) and validatation if the result is consistent.

This becomes even more critical as soon as, in a group, the integration task is performed by a dedicated person. That person, the integrator, does not necessarily know the changes performed and would not be able to solve merge problems, nor it is his/her duty to fix the bugs that can result from the merge. At DS currently, each group has a integrator, and 15 integrators are working full time.

We claim that basic concurrent engineering policies can be defined as the valid sequences of operations performed on objects and attributes in a group. A definition of a few of the most useful policies follows.

Notations. In the following, let us denote by letter P, I, S, R and F commands Propose, Integrate, Synchronize Reserve and Free. C(O.A) denotes one or more change of attribute A; A,B the fact action B does not necessarily follow immediately A, (A1,A2 ... $\mathrm{R})$ the repetition of the sequence in parenthesis if the $\mathrm{R}$ operation fails.

Finaly, letters in Roman caracters are executed by users in a WS, while in italic, they are executed by the integrator (i.e. from the integration WS).

Exclusion. O.A: (SR),C,PIF.

This sequence states the policy to be applied to attribute O.A in a group of WSs. It means that before changing A (C), a WS must first synchronize (S) then reserve (R) A. If the 
reserve fails, the couple SR must be repeated at a later time. After the changes, the WS executes proposes $(\mathrm{P})$, integrates $(\mathrm{I})$ and frees $(\mathrm{F})$ on the object.

This policy implements an ACID transaction ${ }^{4}$ by a two phase locking protocol. S copies the latest official value of the object to the local cache (WS), R sets a lock on the attribute before changing it and thus Integrate simply replaces in the reference repository (Integration WS) the old value by the new one (no merge), and F releases all locks. In practice any attribute for which no merge function exists should be managed in this way.

Delayed reserve: O.A: C,(R)SPIF.

This sequence states that changes on attribute A can be done before reserving it. As a consequence the synchronize operation (S) may require a merge (because meanwhile, a change on A may have been integrated). But since $\mathrm{S}$ is performed in the critical section (i.e. between $\mathrm{R}$ and $\mathrm{F}$ ), operation I (integrate) will never require a merge (because since the reserve, only that WS can integrate a change on A). That policy has the property to never produce any merge in the integration WS. This is a consequence of any sequence in which $\mathrm{S}$ is before $\mathrm{I}$ in a critical section.

\section{Delayed reserve and integration. O.A: C,RSP,I,F.}

This sequence is similar to Delayed reserve, but I and F are performed by the integrator, at integrator convenience. Defered Integration deferred means the integrator is free to select which proposed change to integrate, when and in which order. Since the Free operation can also be delayed, it means the integrator has the opportunity, for example, to run test suites before validating the changes $(\mathrm{F})$. With respect to the previous policy, flexibility is provided to integrators, not to developers.

Note that no merges are ever needed in the Integration WS. In other words, multiple concurrent changes are allowed but their integration is sequentialized and merges are performed in the WS which performed the changes, never in the integration WS. Integrators select the right change to integrate, and users can only synchronize on validated changes $(\mathrm{F})$. This is a flexible but still pretty well controled evolution strategy.

Remember that a policy is defined on an attribute basis. For example, at DS, the management of source code objects in development groups is to use Exclusion for the filename attribute, Delayed reservation and integration for sourceCode attribute and default (no control) for the components attribute.

This approach contrasts with all other SCM cooperation strategies in the following ways:

- A policy can be defined independently for each attribute (default means no

${ }^{4}$ In our implementation, a WS is a (complex) object. Activities performed in a WS can be considered to be a transaction if the S,P,I operations involve the object WS instead of objects it contains. The rollback operation requires creating a revision (deep revision because of the transitive components relationship) of the WS before starting a transaction. This is why, in DS, some WSs have more than 2000 revisions 
control). For instance, in a DS development group, it is possible, for the same object, to have three WS changing the file (sourceCode) another one changing that file name (fileName) and a third one changing which complex object it pertains to (components). Subsequent synchronization will consistently merge all these changes.

- A policy is declared on a group type basis i.e. all WSs in a group share the same policies; but each group can have different policies over the same objects. Typically, the development groups have more relaxed policies than the release group.

- A policy is formally defined (as the valid sequence of commands), and some "classic" policies have been studied so that their properties have been proved and used to optimize the implementation.

The integration WS plays the role of the coordinator for the whole group, it knows the policies and thus enforces and optimizes the controls, based on the properties of each policy.

\subsection{Company Concurrent Work Policies}

The integration WS behaves as the representative of the whole group, and can thus be a component of another higher level group (potentially of another type). In this way, the whole company can be organized in a hierarchy of WSs. At DS, three types of WS are defined, the depth of the WS tree ranges from 2 to 6 , the number of concurrent WSs amounts to several hundred at any point in time. Traditionally, the WSs close to the root are the most stable (release WSs), while the leaf WSs are the most evolutive (Development WSs). Of course different policies apply.

It can be proven that the properties identified inside a single group hold for any sub-hierarchy with the single condition that reserving an attribute in a WS is considered as reserving that attribute in the integration WS of that group. It makes the reserve command transitive between groups which include the reserve command for that attribute in their policy.

The strict application of the previous strategy leads to a tree of groups, with data flow following the edges. Experience have shown this strategy supports the majority of the data flow, but is too limited. Data flow between siblings is needed, as well as data flow between almost any arbitrary group (as long as it is accepted by the process in place). Typical exemples are urgent fix procedures, where a fix "jumps" directly from a low level group to the top of hierarchy; or when a dependent group requires another sibling to make a bloking change.. In both cases the standard procedure would take days, which is sometimes not acceptable.

A major difficulty in SE is that almost any policy sometimes needs to be violated, for "good" reasons. This requires two features: first, the basic mechanisms must be able to perform un- standard operations, and second, these unusual procedures must be carefully defined and closely controlled; this is the goal of general process support, not presented in this paper. 
We have demonstrated that synchronization between siblings is possible, still satisfying the coordination properties, provided some constraints, but at the cost of more expensive algorithms (many optimizations are no longer valid); demonstration is outside the scope of this paper.

Non sibling synchronization is also possible but breaks the coordination properties; for that reason, the underlying mechanisms keeps track of "abnormal" object synchronization (any synchronization that does not follow the tree); because it invalidates the assumptions used for optimization, it is required for performing subsequent merges (e.g. relationship merges require to store information), and is used for history, tracking and debugging.

These "simple" enhancements, as well as the facility allowing to "undo" complex operations like synchronize or integrate are responsible for significantly increasing the complexity of the whole system (remember that these commands can involve very complex objects, often the whole WS, which amounts to thousands of files).

\section{Related Work}

Object management, concurrent Engineering and distributions are hot topics. Nevertheless, it is a surprise to see that not much work have addressed seriously the issues of managing consistently copies of the "same" object having different values, different locations, different definitions and different format.

Databases have addressed the issue of consistency using the transaction concept, and different definitions (schema) with the multi-database and multi-view approaches. Some work has defined sub-databases, which look similar to SCM work spaces, [2][3]. But still there is a single (logical) version and a single consistency approach: serializability.

Middleware deal with object and distribution, but often objects do not move (CORBA $1 \& 2$ ) and in any case objects have a single (logical) value at any point in time. Service on top of Corba propose different coordination (flexible transaction features) and life cycle strategies; nevertheless there is not multiple version management and synchronization facility (yet).

The Web protocols, like HTTP also address some issues, but with different goals : a copy is sent with the assumption it will not be modified. WebDAV is proposing HTTP extensions for "distributed authoring" which includes attributes on objects, complex objects and locks [22][23] . Current proposed extensions to WebDAV [4] include versioning, workspace and configuration support . It is no surprise to note that these extensions are proposed by ClearCase, the leading SCM product [17][18]. Pushing this initiative further could lead to propositions similar to those contained in this paper[8].

Without surprise, SCM systems are those products coming closer to our reauirements. $[6][14][13][11][21]$ but SCM systems, generally, manage files rather than objects, they 
know a single representation (the file) and evolution constraints are missing or unclearly stated[7][12].

\section{Conclusion}

Object management in SCM sets a number of very hard constraints. Our experience shows that the architecture followed by all vendors does not scale enough, does not provide sufficient efficiency, reliability and availability, and does not provide sufficiently high level services for concurrent engineering control. The dramatic increase in product size, concurrency factor and distribution will soon require a complete redesign of these systems.

We advocate for autonomous, heterogeneous and simple WS managers. They provide for the required availability, autonomy and efficiency. Heterogeneity affords for different format and projection conventions, and allows to reuse "standard" tools, preserving habits and investments. It also makes possible the incremental adoption of SCM strategies and policies, as well as scalability capabilities. All this is missing in today's SCM tools.

We claim that concurrent engineering requires the formalization of high level and flexible policies. We have proposed the group concept, and policy definition based on the valid sequence of 5 basic operations. This proposal contributes the idea that a policy is defined on a group basis, for each attribute individually, and may have properties that can be proved, and on which the system relies for performing substantial optimisations. Most policies of interest are available in standard (and recognized for optimisation) and any other can be user defined on the same basis.

Most of the complexity of the system comes from the fact that any policy may need to be violated. We believe it to be of critical importance (1) to define and control the processes where these violations are allowed to occur, and (2) propose a system where this is possible, even at the cost of performance degradation.

We think these propositions really contribute to build system, scalable both in term of amount of managed data, geographical location and level of services provided.

\section{References}

[1] ClearGuide: Product Overview". Technical report, Atria Software, Inc.

[2] A. Bjornersledl and C. Hullen. Version control in an Object-Oriented Architecture. In Won Kim and Frederick H. Lochowsky. editors. Objects-Oriented concepts, databases and application. Chapter 18, pages 451-485, Adisson-Wesley. 1990.

[3] E. Bratsberg. Unified Class Evolution by Object Oriented views. Proc of the 11th Conf on the relationship approach. LNCS N0645, Springer Verlag, Oct 1992.

[4] G. Clemm. Versioning Extensions to WebDav. Rational Software. May 1999. http:// www.ietf.org/internet-drafts/draft-ietf-webdav-versioning-02.txt 
[5] G. Clemm. The Odin System. SCM5, Seattle June 1995, pp241-263. Springer Verlag LNCS 1005.

[6] S. Dami, J. Estublier and M. Amiour. "APEL: a Graphical Yet Executable Formalism for Process Modeling". Automated Software Engineering journal, January 1998.

[7] S. Dart. "Concepts in Configuration Management Systems". Proc. of the 3rd. Intl. Workshop on Software Configuration Management. Trondheim, Norway, june, 1991.

[8] S. Dart. Content Change Management: Problems for Web Systems. In Proc SCM9, Toulouse, France, September 1999. pp1-17. Springer Verlag LNCS 1675.

[9] J. Estublier and R. Casallas. "The Adele Software Configuration Manager". Configuration Management, Edited by W. Tichy; J. Wiley and Sons. 1994. Trends in software.

[10] J. Estublier and R. Casallas. "Three Dimensional Versioning". In SCM-4 and SCM-5 Workshops. J. Estublier editor, September, 1995. Springer LNCS 1005, pp118-136.

[11] J. Estublier. "Workspace Management in Software Engineering Environments". in SCM6 Workshop. Springer LNCS 1167. Berlin, Germany, March 1996.

[12] J. Estublier and S. Dami and M. Amiour. High Level Process Modeling for SCM Systems. SCM 7, LNCS 1235. pages 81--98, May, Boston, USA, 1997

[13] J. Estublier and R. Casallas. "Three Dimensional Versioning". In SCM-4 and SCM-5 Workshops. J. Estublier editor, September, 1995. Springer LNCS 1005.

[14] B. Gulla, E.A. Carlson, D. Yeh. Change-Oriented version description in EPOS. Software Engineering Journal, 6(6):378-386, Nov 1991.

[15] B. Gulla, J. Gorman. Experience with the use of a Configuration Language. In SCM-6 Workshop, Berlin, Germany, March, 1996. Springer Verlag LNCS1167, pp198-219.

[16] M. Hardwick, B.R. Dowine, M. Kutcher, D.L. Spooner, "Concurrent Engineering with Delta Files', IEEE Computer Graphics and Applications, January 1995, pp. 62-68.

[17] D. B. Leblang. and G.D. McLean. Configuration Management for large-scale software development efforts. In Proceedings of the workshop on Software Environments for Programming-in-the-Large. Pages 122-127. Harwichport, Massachussets, Jume 1985.

[18] D. B. Leblang. "The CM Challenge: Configuration Management that Works". Configuration Management, Edited by W. Tichy; J. Wiley and Sons. 1994. Trends in software.

[19] D.B. Leblang. Managing the Software Development Process with ClearGuide. SCM 7 , LNCS 1235. pages $=66,80$, May, Boston, USA, 1997

[20] J. Micallef and G. M. Clemm. "The Asgard System: Activity-Based Configuration Management". In SCM-6 Workshop, Berlin, Germany, March, 1996. Springer Verlag LNCS1167, pp175-187.

[21] Walter F. Tichy. Tools for software configuration management. In Proc. of the Int. Workshop on Software Version and Configuration Control, pp. 1-20, Grassau, January 1988

[22] Jim Whitehead. Goals for a Configuration Management Network protocol.In SCM9, LNCS 1675, pages 186-204, Toulouse September 1999.

[23] WebDav. HTTP extentions for distributed Authoring. RFC 2518. http:// andrew2.andrew.cmu.edu/rfc/rfc2518.htm. February 1999. 